\title{
Temperature of maximum density and excess properties of short-chain alcohol aqueous solutions: A simplified model simulation study
}

A. P. Furlan, E. Lomba, and M. C. Barbosa

Citation: The Journal of Chemical Physics 146, 144503 (2017); doi: 10.1063/1.4979806

View online: https://doi.org/10.1063/1.4979806

View Table of Contents: http://aip.scitation.org/toc/jcp/146/14

Published by the American Institute of Physics

\section{Articles you may be interested in}

Perspective: Dissipative particle dynamics

The Journal of Chemical Physics 146, 150901 (2017); 10.1063/1.4979514

Statistical properties of fluctuating enzymes with dynamic cooperativity using a first passage time distribution formalism

The Journal of Chemical Physics 146, 145103 (2017); 10.1063/1.4979945

A Gaussian theory for fluctuations in simple liquids

The Journal of Chemical Physics 146, 134507 (2017); 10.1063/1.4979659

Water/ice phase transition: The role of zirconium acetate, a compound with ice-shaping properties

The Journal of Chemical Physics 146, 144504 (2017); 10.1063/1.4979845

Communication: Mean-field theory of water-water correlations in electrolyte solutions

The Journal of Chemical Physics 146, 181103 (2017); 10.1063/1.4983221

Anomalous sound attenuation in Voronoi liquid

The Journal of Chemical Physics 146, 144502 (2017); 10.1063/1.4979720

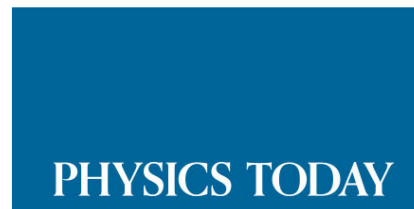




\title{
Temperature of maximum density and excess properties of short-chain alcohol aqueous solutions: A simplified model simulation study
}

\author{
A. P. Furlan, ${ }^{1}$ E. Lomba, ${ }^{2}$ and M. C. Barbosa ${ }^{1}$ \\ ${ }^{1}$ Instituto de Física, Univeridade Federal do Rio Grande do Sul, Caixa Postal 15051, 91501-570 Porto Alegre, \\ Rio Grande do Sul, Brazil \\ ${ }^{2}$ Instituto de Química Física Rocasolano, CSIC, Serrano 119, E-28006 Madrid, Spain
}

(Received 31 January 2017; accepted 22 March 2017; published online 12 April 2017)

\begin{abstract}
We perform an extensive computational study of binary mixtures of water and short-chain alcohols resorting to two-scale potential models to account for the singularities of hydrogen bonded liquids. Water molecules are represented by a well studied core softened potential which is known to qualitatively account for a large number of water's characteristic anomalies. Along the same lines, alcohol molecules are idealized by dimers in which the hydroxyl groups interact with each other and with water with a core softened potential as well. Interactions involving non-polar groups are all deemed purely repulsive. We find that the qualitative behavior of excess properties (excess volume, enthalpy, and constant pressure heat capacity) agrees with that found experimentally for alcohols such as t-butanol in water. Moreover, we observe that our simple solute under certain conditions acts as a "structuremaker," in the sense that the temperature of maximum density of the bulk water model increases as the solute is added, i.e., the anomalous behavior of the solvent is enhanced by the solute. Published by AIP Publishing. [http://dx.doi.org/10.1063/1.4979806]
\end{abstract}

\section{INTRODUCTION}

Processes involving mixtures of water and a variety of organic compounds are present in a huge diversity of phenomena. In most cases, effects of hydrogen bonding and hydrophobicity are the key elements in determining the behavior of such mixtures. These range from the simplest case of diluted short chain alcohols to the substantially more involved situations of biomolecules (e.g., proteins) in solution. The former have attracted special attention from the technological standpoint due to their relevance in the bioethanol industry ${ }^{1,2}$ as well as in pharmaceutical and cosmetic industries, being some of the preferred solvents for a wide range of solutes with varying degrees of polarity. Moreover, from a fundamental perspective the study of dilute short chain alcohol solutions is of utmost importance, being the simplest systems that illustrate the interplay of hydrogen bonding and hydrophobicity in amphiphilic substances. In particular, their thermodynamics is known to exhibit quite a few characteristic features, such as the presence of maxima in the excess specific heat, ${ }^{3,4}$ minima in the excess volume, ${ }^{3,5}$ and negative excess entropy. ${ }^{6}$ Some of the anomalies found in these systems are in close connection with more than seventy anomalies present in water, among them, the presence of a density maximum at $3.98 \mathrm{C}$ and $1 \mathrm{bar}$ in liquid water. ${ }^{7}$

The anomalous behavior of water in the fluid phase has been explained in terms of the competition between a low density structure dominated by the presence of hydrogen bonds and exhibiting essentially tetrahedral ordering and a high density one, with higher coordination and a much lower degree of hydrogen bonding. For temperatures above the temperature of maximum density (TMD), the high density structure dominates, and the system expands upon heating, whereas below the TMD the loosely coordinated structure is the preferred one and the system contracts upon heating, due to the breakup of the hydrogen bond network.

The way in which solutes modify the anomalous properties of water is not yet completely understood. In this respect, concerning the density anomaly, solutes can be classified into two groups, namely "structure-makers" (as they increase the TMD when added to water) and "structure-breakers" (decrease the TMD). ${ }^{8,9}$ It has been found that solutes whose molecules do not join the hydrogen bond network, such as electrolytes, ${ }^{10}$ room temperature ionic liquids, ${ }^{11}$ or polar substances without $\mathrm{H}$-bond active groups ${ }^{12}$ (e.g., acetone, acetonitrile, tetrahydrofuran, among others), induce a decrease of the TMD. They can all be cast into the group of "structure-breakers." In contrast, dilute solutions of hydrogen bond forming substances with relatively small non-polar tails, such as short-chain alcohols ${ }^{13}$ and some amines, ${ }^{14}$ exhibit an increase of the TMD with respect to pure water. These substances are thought to enhance the structuring of the tetrahedral low density phase of water, and thus are "structure-makers." The change in the TMD is measured in terms of $\Delta T_{M D}=T_{M D}\left(x_{2}\right)-T_{M D}\left(x_{2}=0\right)$, where $T_{M D}\left(x_{2}\right)$ represents the temperature of maximum density of the solution at a given molar fraction of the solute, $x_{2}$, and $T_{M D}\left(x_{2}\right.$ $=0$ ) obviously refers to the TMD of pure water. Typically, for a structure-maker $\Delta T_{M D}>0$ until a given solute mole fraction for which a maximum is reached, and then it decreases up to a certain concentration where the solution no longer presents a maximum density in terms of temperature. From the work of Wada and Umeda, ${ }^{13}$ it was found that the largest increase in the TMD at atmospheric pressure occurs for t-butanol at $x_{2} \simeq 0.0043$ with $\Delta T_{M D}=0.41 \mathrm{~K}$.

In close connection with the anomalies found in dilute hydrogen-bonding water solutions, the excess mixture 
properties are also known to exhibit certain singularities. Thus, for instance, in the case of small linear chain alcohols (methanol ${ }^{15,16}$ and ethanol ${ }^{15,17}$ ) and alkylamines, ${ }^{18}$ the excess enthalpy is negative, whereas for somewhat larger non-polar tails (propanol and butanol), this same property changes its concavity and assumes positive values. Ionic liquids behave differently depending on the degree of hydrophobicity of their non-polar tails: less hydrophobic ionic liquids have a negative excess mixture enthalpy, whereas hydrophobic ionic liquids display positive excess enthalpies of mixing with a maximum. The excess mixture volume at ambient conditions is negative and exhibits a minimum for alcohols s,19-21 $^{5}$ and alkylamines. ${ }^{22,23}$ Similarly to the excess enthalpy, the excess volume in ionic liquid aqueous solutions depends on the degree of hydrophobicity of the solute. ${ }^{24}$ The excess specific heat displays a peculiar behavior for small alcohol concentrations, e.g., in water-methanol mixtures a maximum occurs at solute concentration $x_{\mathrm{MeOH}}=0.16^{4}$ and for t-butanol ${ }^{25}$ for $x_{t-\mathrm{BuOH}} \approx 0.08$.

The first attempt to explain the excess thermodynamics of amphiphilic aqueous solutions dates back to 1945, with the pioneering work of Frank and Evans. ${ }^{26}$ They established a connection between structure and the thermodynamics of mixtures and formulated the "iceberg theory." According to this interpretation, the presence of the composition-dependent anomalies can be ascribed to the formation of a low entropy cage of water with strong hydrogen-bonds around the alcohol molecules, which in this case would increase the structural ordering of bulk water. These ideas seem to have been confirmed by a series of experimental X-ray diffraction studies, in most cases complemented by molecular simulations. ${ }^{27,28}$ In these studies, it was found that adding methanol to water enhances the local three-dimensional network of water in the vicinity of the methanol molecules, which explains the decrease in the entropy of mixing and the considerable increase in the heat capacity at low alcohol concentrations.

In an attempt to provide a more quantitative analysis, Chatterjee et al $^{29}$ resorted to a simple statistical mechanical model and ascribed the increase in the TMD to the hydrophobicity of the solute (the non-polar tail). Within their approach, the dispersive solute-solvent interactions are thought to be responsible for the decrease of the density anomaly to lower temperatures. Their picture, however, downplays the role that hydrogen bonding must necessarily play in the phenomenology of alcohol (or alkylamine)-water mixtures and the essentially different behavior of other polar solutes, such as acetonitrile or acetone. ${ }^{12}$ Somewhat more sophisticated models have been developed in which the alcohol is represented using site-site molecular models (in the simplest case of methanol, a dimer), and the hydrogen bonding interactions are modeled using a two-scale potential, both of a core softened type $^{30-33}$ and a Jagla type ${ }^{34}$ ramp potential. ${ }^{35}$ These models give rise to features, such as liquid-liquid equilibria ${ }^{31}$ or the presence of a TMD curve in methanol, which have not been confirmed experimentally. On the other hand, these models can be fitted to reproduce qualitatively the behavior of the excess mixture properties. ${ }^{35}$ In none of these cases, the influence of alcohol on the change of the TMD of water has been reproduced.
More sophisticated models can be tackled resorting to computer simulation. In these cases, the mixture has been modeled using all-atom site-site interaction potentials, such as TIP4P/2005 for water ${ }^{36}$ or Optimized Potentials for Liquid Simulations (OPLS) for alcohols. ${ }^{37}$ Concerning structural features of alcohol-water mixtures, Allison et.al. ${ }^{38}$ showed using molecular dynamics that the number of hydrogen bonds decreases and the water molecules become distributed in rings and clusters as the alcohol concentration increases, in accordance with experimental results. ${ }^{6}$ The simulation results of Laaksonen et al. ${ }^{39}$ indicated that the system is highly ordered around the hydroxyl groups, and methanol molecules are solvated by water molecules, in accordance with the assumptions of iceberg theory. ${ }^{3,26}$ Also using molecular dynamics simulations, Bako and co-workers showed that despite the decreasing number of hydrogen bonds in the mixture, the tetrahedral structure of water is preserved. ${ }^{40} \mathrm{~A}$ recent mixture model for methanol-water developed by GonzálezSalgado and co-workers ${ }^{41}$ has shown to be able to reproduce quantitatively the excess thermodynamic properties, but does not account for the "structure-maker" character of methanol molecules at high dilution, and in fact the decrease in the TMD with concentration predicted by the model is practically one order of magnitude larger than the experimental.

It is then clear that there is much room for improvement in our knowledge of the dilute solutions of hydrogen bonding substances in water. In this work, we aim at obtaining further insight using continuous site-site two-scale potential models, which are simple enough to discriminate the different effects than enter the structural and thermodynamic behavior of the model, but at the same time are able to reflect the anomalous features of water-like systems. To that aim, we have studied here the excess thermodynamics and the density anomaly of a mixture of water and amphiphilic dimers, in which water (solvent) is represented by a spherically symmetric two length scale potential, ${ }^{42}$ and the alcohol molecules (dimers) are modeled by a repulsive $\mathrm{R}$-site and an $\mathrm{OH}$-site which interacts with other $\mathrm{OH}$ sites and with water by means of a two length scale potential. This system will be studied by means of extensive molecular dynamics calculations in various ensembles, with different two sizes of the apolar site of the alcohol-like molecule, a methanol-like model (homonuclear) and a tert-butanol-like one (heteronuclear), in which the R-site is substantially larger than the $\mathrm{OH}$-site.

The remaining of the paper is organized as follows. In Section II we present our models for water and alcohol molecules. In Section III relevant technical details of the simulations are presented. Next, in Section IV the results for our hetero- and homo-nuclear alcohol models in solution are introduced, both concerning excess properties and influence on the TMD of water. A brief summary and a presentation of our main conclusions and future prospects close this article in Section V.

\section{THE MODEL}

As mentioned above, we will have spherical particles representing water-like molecules, together with an amphiphilic solute with a purely repulsive site accounting for the apolar 
tail, $\mathrm{R}$, in addition to an $\mathrm{OH}$ site, characterized by $\mathrm{OH}-\mathrm{OH}$ and $\mathrm{OH}$-water interactions with two length scales. ${ }^{42} \mathrm{~A}$ short range repulsion accounts for the high density liquid phase, and a much longer range repulsion and attraction attempt to roughly model the more open structures due to hydrogen bonding. To make matters simpler, we will use the same softened-core potential for water-water, $\mathrm{OH}-\mathrm{OH}$, and $\mathrm{OH}-$ water interactions, defined by

$$
\begin{aligned}
U_{s c}\left(r_{i j}\right)= & 4 \epsilon_{s c}\left[\left(\frac{\sigma}{r_{i j}}\right)^{12}-\left(\frac{\sigma}{r_{i j}}\right)^{6}\right] \\
& +\sum_{\ell=0}^{1} u_{\ell} \epsilon_{s c} \exp \left[-\frac{1}{c_{\ell}^{2}}\left(\frac{r_{i j}-r_{\ell}}{\sigma}\right)^{2}\right] .
\end{aligned}
$$

Here, $r_{i j}$ represents the separation between sites $i$ and $j$. The first term on the rhs of Equation (1) is the standard 12-6 Lennard-Jones (LJ) potential, ${ }^{43}$ whereas the second term is the summation of two Gaussians, centered at $r_{0}=0.7 \sigma$ and $r_{1}=3 \sigma$, with depths $u_{o}=5$ and $u_{1}=-0.75$ and widths $c_{0}=1$ and $c_{1}=0.5$, respectively. The potential of Eq. (1) displays two different length scales, an attractive scale at $r \approx 3 \sigma$ and a repulsive shoulder at $r \approx \sigma$. Of the many possible choices of two-scale potentials, ours has been motivated by its ability to account for many of the anomalous features of fluid water, ${ }^{42,44,45}$ displaying the characteristic cascade ordering of anomalies. ${ }^{46}$ For the parameters chosen in this work, the model is known to display a density anomaly with a TMD curve in the supercritical region. ${ }^{42}$ The attractive well that can be seen in Figure 1 is not sufficient to place the anomalous region within the stable liquid phase, in contrast with the situation in real water. Despite these limitations, as already mentioned, this model potential is an excellent candidate to reproduce water anomalies. $^{42}$

The non-polar site-site interactions ( $\mathrm{R}-\mathrm{R}, \mathrm{R}-\mathrm{OH}$, and Rwater) are represented by a purely repulsive Weeks-ChandlerAndersen (WCA) potential ${ }^{47}$ of the form

$$
U_{r}\left(r_{i j}\right)= \begin{cases}U_{L J}\left(r_{i j}\right)-U_{L J}\left(r_{c}\right) & \text { if } r \leq r_{m}, \\ 0 & \text { if } r>r_{m},\end{cases}
$$

where $U_{L J}(r)$ is the standard 12-6 LJ potential with parameters, $\epsilon_{r}, \sigma_{r}$, and $U_{L J}\left(r_{m}\right)$ is the LJ potential computed at a

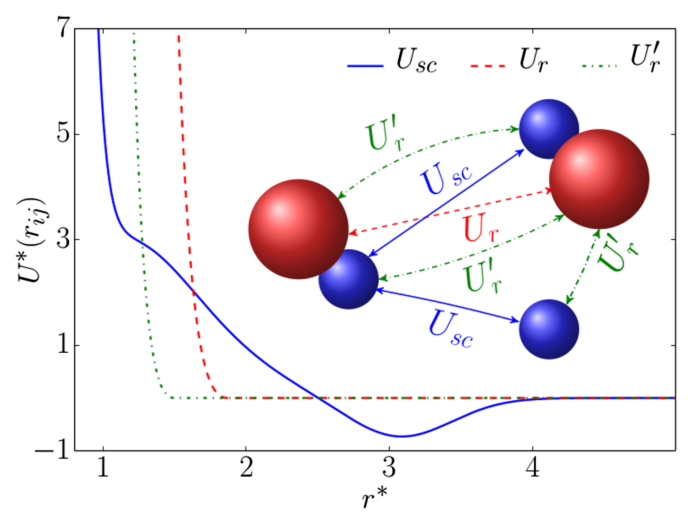

FIG. 1. Interaction potential versus distance. The solid blue line represents the softened-core interaction potential $U_{s c}$ (Equation (1)) between $\mathrm{OH}-\mathrm{OH}$, $\mathrm{OH}$-water, and water-water sites. The dashed red line and green dotted-dashed line represent the $\mathrm{R}-\mathrm{R}, \mathrm{R}-\mathrm{OH}$, and $\mathrm{R}-$ water repulsive interactions. cutoff distance given by the position of the minimum of the LJ interaction, $r_{m}=2^{1 / 6} \sigma_{r}$.

In what follows, we have used as unit length, $\sigma=\sigma_{w w}=\sigma_{w-\mathrm{OH}}=\sigma_{\mathrm{OH}-\mathrm{OH}}$, and as energy unit, $\epsilon_{s c}$. Reduced pressure and temperature are defined as $P^{*}=P \sigma^{3} / \epsilon_{s c}$ and $T^{*}=k_{B} T / \epsilon_{s c}$, where $k_{B}$ is Boltzmann's constant. The simulation time step is given in reduced units of $\tau=\sigma \sqrt{m / \epsilon_{s c}}$, where $m$ is one of the particle masses. Since here we are not interested in dynamic properties, we have considered all particle masses identical.

As mentioned, we have considered a heteronuclear model, in which $\sigma_{r} / \sigma=5 / 3$ (a rough model for tert-butanol), and a homonuclear model in which $\sigma_{r} / \sigma=1$.

The energy parameter of the repulsive interaction was set to $\epsilon_{r} / \epsilon_{S c}=1.21$ and the dimer bond length to $d_{R-\mathrm{OH}}$ $=0.48 \sigma$. This choice of parameters was to some extent inspired by the OPLS force field widely used to simulate alcohols. ${ }^{37}$ Cross interaction parameters were computed using Lorentz-Berthelot mixing rules. ${ }^{43}$ A graphical representation of our molecular models and the corresponding interactions is depicted in Figure 1.

\section{SIMULATION DETAILS}

Using the LAMMPS package, ${ }^{48}$ we have performed MD simulations for a system with a number of particles ranging from 2000 to 4000 for various compositions.

The simulations were performed in the NPT ensemble with a Nosé-Hoover thermostat and barostat ${ }^{49,50}$ and particles were placed in a cubic box with the standard periodic boundary condition. The dimer bonds were kept fixed using a SHAKE algorithm, ${ }^{51}$ with a tolerance factor of $10^{-5}$. Since the system can undergo a demixing transition, we have systematically checked that the thermodynamic conditions under consideration were away from instability by the inspection of the small wave vector behavior of the concentration-concentration structure factor. ${ }^{52,53}$ For our mixture, this quantity is defined by

$$
\begin{aligned}
S_{c c}(Q)= & x_{R \mathrm{OH}}^{2} S_{w w}(Q)+x_{w}^{2} S_{R \mathrm{OH}-R \mathrm{OH}}(Q) \\
& -2 x_{R \mathrm{OH}} x_{w} S_{w-R \mathrm{OH}}(Q),
\end{aligned}
$$

where $x_{w}$ and $x_{R \mathrm{OH}}=1-x_{w}$ are the mole fractions of water and alcohol, respectively. For the partial structure factors, we have approximated $S_{R \mathrm{OH}-R \mathrm{OH}}=S_{R R}$ and $S_{w-R \mathrm{OH}}=S_{w R}$, i.e., we have neglected the contribution of the $\mathrm{OH}$-sites of the dimer. In the study of demixing, this approximation is harmless, since the positions of $\mathrm{R}$ and $\mathrm{OH}$ sites within the same molecule are obviously tightly bound. The site-site structure factors are numerically determined from the spatial configurations generated during additional NVT simulation runs (in order to keep the box size constant for the binning procedure in Q-space) using standard procedures. ${ }^{53}$

The signature of concentration fluctuations associated with demixing is typically a low-Q diverging concentrationconcentration structure factor. By monitoring this quantity along our simulations, we have ruled out the presence of inhomogeneities due to demixing. 
Our simulations started from a compositionally disordered mixture of $\mathrm{ROH}$ and water particles, which was equilibrated at the chosen pressure and temperature for $1 \times 10^{7}$ steps in the NPT-ensemble. Production runs were $8 \times 10^{7}$ step long. The time step was set to $5 \times 10^{-6} \tau$ in reduced units.

\section{RESULTS}

In what follows, we will present our results both for the hetero- and homo-nuclear ROH models in a solution of our water-like fluid, first focusing on the ROH influence on the temperature of the maximum density curve of water (which was already determined in Ref. 42). We will analyze the influence of the alkyl-group size on the changes of the TMD, comparing the results of our heteronuclear and homonuclear models. Finally, we will analyze the behavior of the excess thermodynamics of the mixture just for the heteronuclear model.

\section{A. The temperature of maximum density (TMD)}

The density anomaly in water and $\mathrm{ROH}$ aqueous solutions can be easily detected representing the temperature dependence of the density along isobars. This can be done studying a series of state points along various isobars by means of NPT simulations. These results are presented in Figure 2 for various ROH mole fractions, namely, $x_{R \mathrm{OH}}=0.00,0.01, \ldots, 0.04$, first for our heteronuclear model. Note that the apparent low values of the reduced density are due to the fact that densities are scaled with the inner core of the potential. If scaled with the range of the second repulsive range $(\approx 2.5 \sigma)$, which is a more appropriate measure of the molecular size, we will have reduced densities in line with what one should expect for a liquid $\left(\rho^{*} \approx 0.5 \sim 0.9\right)$.
At a certain concentration of $\mathrm{ROH}$ the TMD disappears, since our ROH model lacks a density anomaly. A relatively accurate numerical estimate of the TMDs was obtained by a polynomial fit to the simulated densities. These points (denoted by solid squares) are connected in Figure 2 with short-dashed lines, that constitute the TMD curve in the $T-\rho$ plane. We observe that the region on the left of the TMD points is characterized by the typical density anomaly, namely, a density increase upon heating. Note that for all compositions the TMD increases with pressure to reach a maximum and then decreases. This decrease of the TMD with pressure corresponds to the experimental behavior found in water ${ }^{54}$ and is the result of the destructuring effect of pressure on the open structures (hydrogen bonded network in the case of water) whose interplay with the high density phase gives rise to the density anomaly. The increase of the TMD with pressure at low pressures is not found experimentally, and it is a consequence of the fact that in our model the TMD curve is placed in the supercritical region. This feature is present even in models for which the TMD curve is in a low density liquid region, such as the ramp fluid. ${ }^{55}$

The various TMD curves for different mole fractions are represented in Figure 3. One readily appreciates that the addition of alcohol reduces the density range and the temperature at which the density anomaly is found, ultimately leading to its disappearance. Points at equal pressure are connected by dashed lines.

The change in the TMD with respect to that of pure water $\left(\Delta T_{M D}\left(x_{R \mathrm{OH}}\right)=T_{M D}\left(x_{R \mathrm{OH}}\right)-T_{M D}\left(x_{R \mathrm{OH}}=0\right)\right)$ induced by the presence of the solute is represented in Figure 4 for various pressures. For pressures below $P^{*} \approx 10$, and up to a certain concentration, we observe that our solute acts as a "structure-maker." This means that the presence of solute molecules enhances the anomalous behavior of water, by favoring the build up of open structures and hence increasing
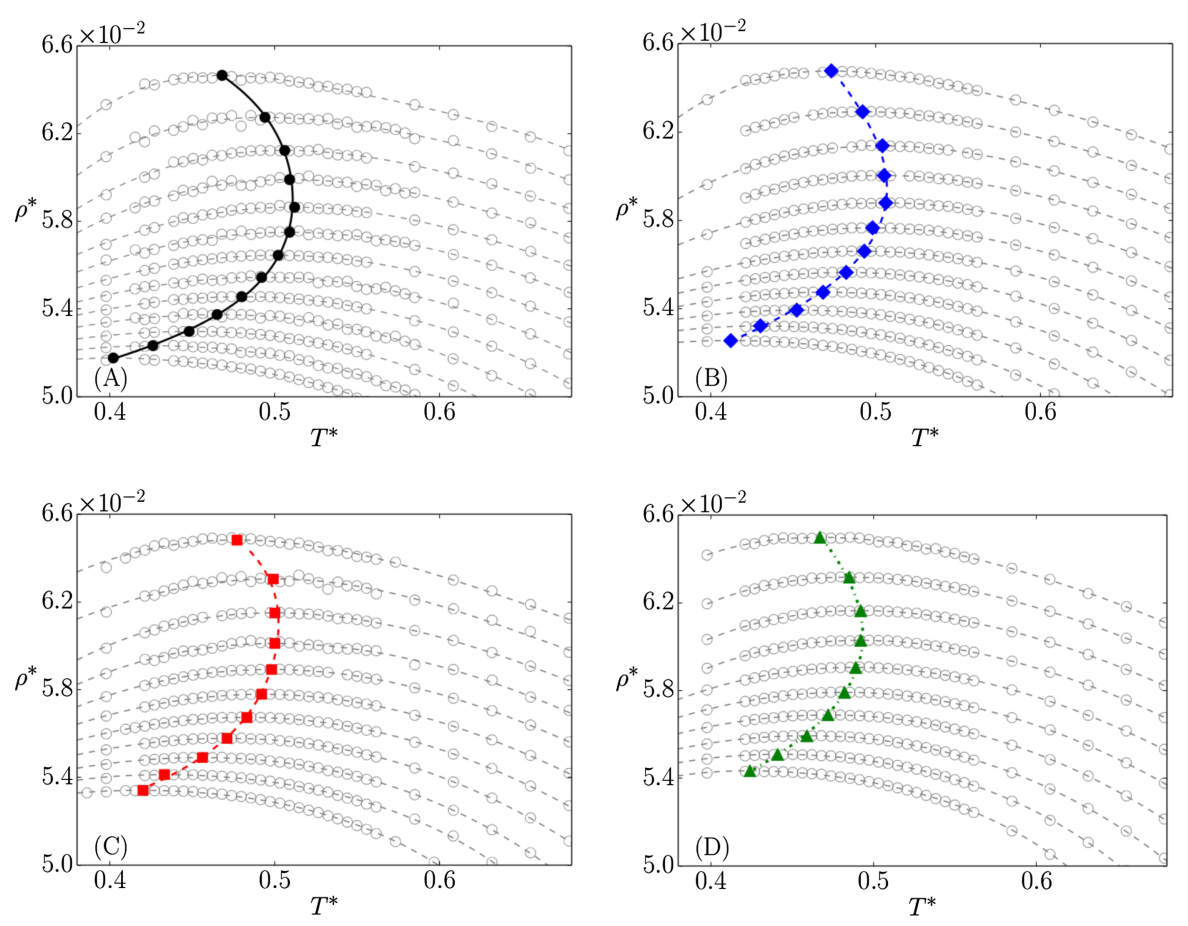

FIG. 2. Temperature dependence of the density for various solute compositions along isobars with increasing pressure from bottom to top $\left(P^{*}=2.3, \ldots, 27.6\right)$. Open circles correspond to simulation data and dashed lines denote a polynomial fit. (a) $x_{R \mathrm{OH}}$ $=0.0$ (pure water), (b) $x_{R \mathrm{OH}}=0.01$, (c) $x_{R \mathrm{OH}}=0.02$, and (d) $x_{R \mathrm{OH}}=0.03$. The TMD is represented by filled symbols, which are connected with a curve that corresponds to a polynomial fit, to represent the TMD curve. Pressure increases from bottom to top. 


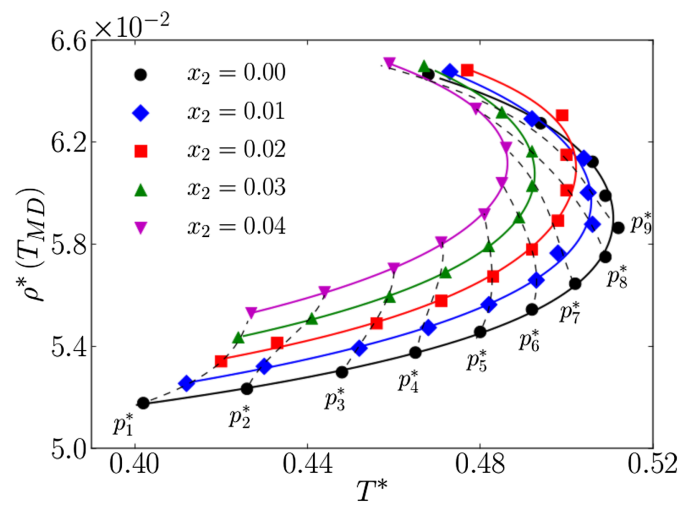

FIG. 3. Density values at the temperature of maximum density of the heteronuclear alcohol model, for various mole fractions, $x_{R \mathrm{OH}}=0.00$ (black solid line and filled circles), $x_{R \mathrm{OH}}=0.01$ (blue line and filled lozenges), $x_{R \mathrm{OH}}$ $=0.02$ (red solid line and filled squares), $x_{R \mathrm{OH}}=0.03$ (green solid line and filled triangles), and $x_{R O H}=0.04$ (magenta solid lines and filled upside down triangles). In all cases, the points are simulation data, and lines correspond to polynomial fits. State points at the same pressure are connected with dashed lines.

the TMD. At $P^{*}=9.2$, the curve presents a maximum around $x_{R \mathrm{OH}}=0.03$ and then decays, which is the qualitative behavior of the TMD tert-butanol in water. ${ }^{13}$ We find that as the pressure increases the change in the TMD is lowered, and as a matter of fact for $P^{*}>10, \Delta T_{M D}\left(x_{R O H}\right)<0$, and the solute behaves as a "structure-breaker," reducing the range of the anomalous behavior of water. This is accordance with the fact that the increase of pressure tends to destroy the low density structures that give rise to the density anomaly, therefore the structuring effect of the solute decreases, to finally turn the "structure-maker" into a "structure-breaker." For sufficiently high pressures, our alcohol-like molecules behave like standard solutes which tend to decrease the TMD, ${ }^{12}$ i.e., the effect of the two-scale interaction stemming from the $\mathrm{OH}$ site is no longer apparent for sufficiently high pressures. A parallel situation occurs with the effect of the hydrogen bonds in water when pressure starts to break them.

Now in Figure 5 we present the corresponding $\rho-T$ TMD curves for the homonuclear model of alcohol in solution. The first effect one can observe is the shift of the TMD curves as a function of the solute concentration is minimized with respect to that observed in Figure 3 for the heteronuclear case. This is a clear indication that the larger the size of the apolar tail of the

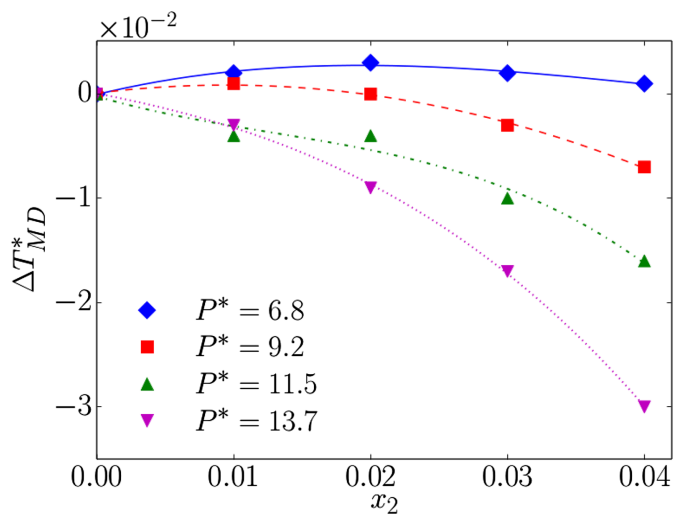

FIG. 4. Change in the temperature of maximum density with respect to the bulk solvent value vs. alcohol mole fraction for various pressures for the heteronuclear alcohol model.

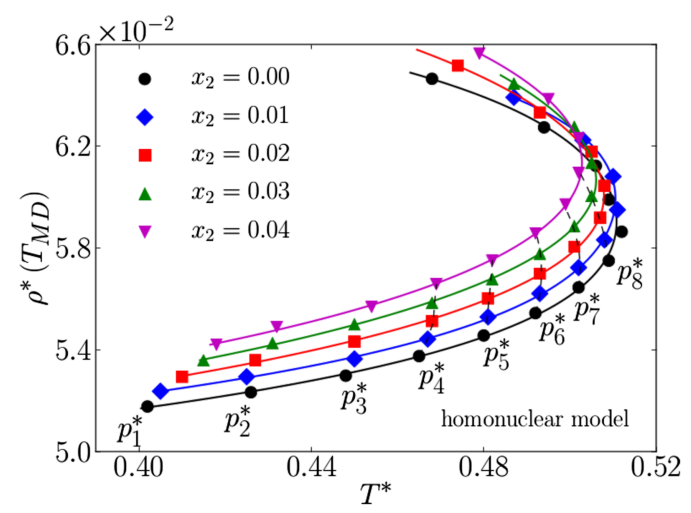

FIG. 5. The same as Figure 3 for the homonuclear alcohol model.

$\mathrm{ROH}$, the more significant the effect of the solute on the TMD. The size dependency of the anomalous behavior is more clearly illustrated in Figure 6, where change in the TMD, $\Delta T_{M D}\left(x_{R \mathrm{OH}}\right)$, for the homonuclear model is represented as a function of alcohol concentration, $x_{R \mathrm{OH}}$ for various pressures. Note that the same scale as in Figure 4 is used. The comparison of both figures shows that the increase in the size of the apolar tail of the alcohol increases the changes in the TMD. On the one hand, for pressures below $P^{*} \approx 10$ the maximum in $\Delta T_{M D}\left(x_{R O H}\right)$ (a characteristic of t-butanol and ethanol in dilution ${ }^{13}$ ) practically disappears for the homonuclear model. Interestingly, this model displays a behavior resembling that of methanol, ${ }^{13}$ for which the maximum is hardly visible. For these values of the pressure, the "structure-maker" character of the model alcohol is enhanced when the apolar chain is larger. This is in agreement with the experimental data and with the theoretical predictions of Chatterjee et al. ${ }^{29}$ statistical mechanical model for solutions of apolar solvents in water. Now, as the pressure increases above, $P^{*} \approx 10$, the solute behaves as a "structure breaker," and interestingly, its effect on $T_{M D}$ is also more significant as the size of the R-site increases to the point that the drop of the TMD for the largest concentration considered is three times larger for the heteronuclear model. Unfortunately, we are not aware of any experimental investigation of the pressure dependence of $\Delta T_{M D}\left(x_{R \mathrm{OH}}\right)$, but since the net effect of pressure is to reduce the range of the anomalous behavior (in real fluids by breaking the hydrogen bond network and in our model by displacing particles towards the first range of the potential),

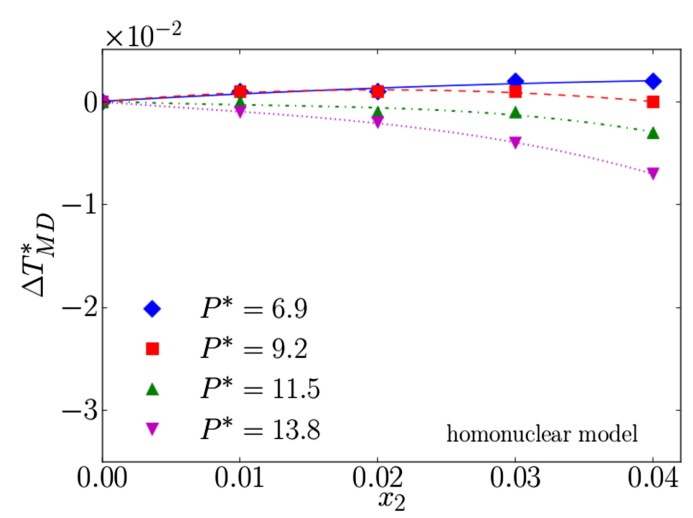

FIG. 6. Change in the temperature of maximum density with respect to the bulk solvent value vs. alcohol mole fraction for various pressures for the homonuclear alcohol model. 

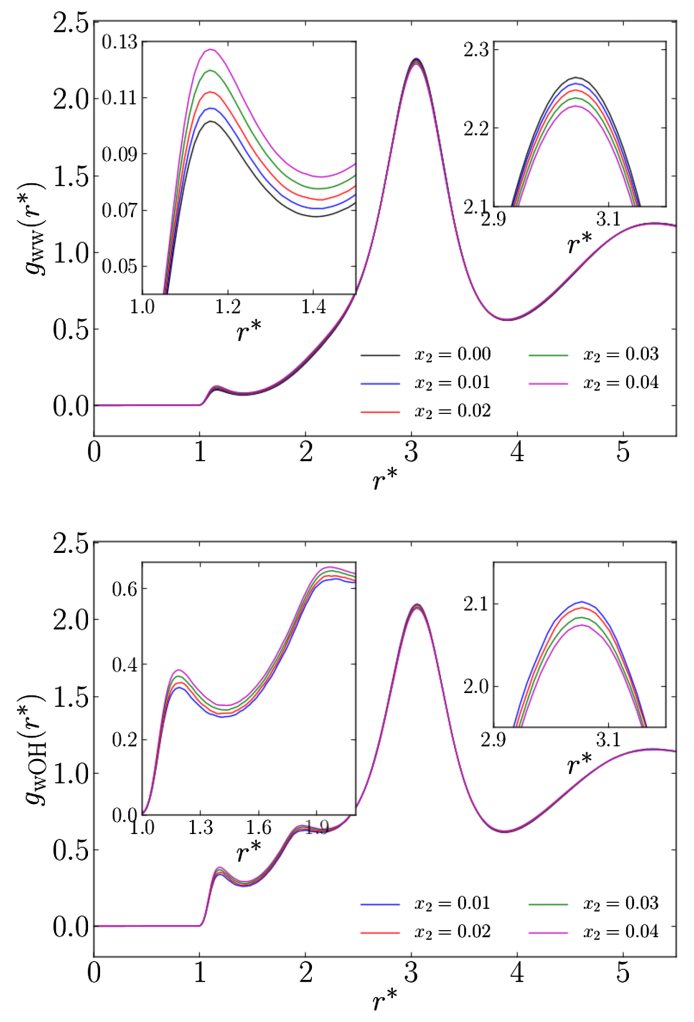

FIG. 7. Water-water (up) and water-OH (down) radial distribution functions for $P^{*}=6.8$ and $T^{*}=0.4$ for various solute concentrations. The insets show a zoom of the regions around the first two maxima.

the fact that the effect is maximized when the volume of the solute is larger is understandable from an enthalpic point of view.

From a microscopic point of view, structural effects of the addition of the solute should be visible in the water-water and water-OH pair distribution functions. These are plotted in Figure 7 for $P^{*}=6.8$ and $T^{*}=0.4$. One observes that a small number water particles move into the first scale of the potential (more compact structures), but at the same time, the area corresponding to the second repulsive range of the potential $(r \approx 2 \sigma)$ becomes more populated, which is particularly visible in the evolution of the second maximum of the $g_{w \mathrm{OH}}$ site-site function. In this way, the addition of solute molecules leads to an increase of open structures and more compact ones. The balance between these open and compact structures is correlated with the subtle change from $\Delta T_{M D}>0$ to $\Delta T_{M D}<0$ as $x_{R \mathrm{OH}}$ grows.

\section{B. Excess thermodynamic properties}

Excess thermodynamic properties of a mixture are defined as the difference between the values of a given thermodynamic quantity and those that would be obtained in an ideal mixture. For a given quantity, $A$, the corresponding excess property is defined by

$A^{E}\left(x_{2}, p, T,\right)=A\left(x_{2}, p, T\right)-\left[x_{2} A_{2}^{0}(p, T)+\left(1-x_{2}\right) A_{1}^{0}(p, T)\right]$,

where $A\left(x_{2}, p, T\right)$ is the value $A$ in the binary mixture of a given composition defined by mole fractions $\left(x_{1}, x_{2}\right) . A_{1}^{0}$ and $A_{2}^{0}$ are the values of $A$ for the pure components at the same thermodynamic state. Quantities of interest in binary mixtures are the excess volume, $V^{E}$, enthalpy, $H^{E}$, and specific heat at constant pressure $c_{P}^{E}$. Excess entropy is also of interest, but it is not directly accessible in MD calculations. Excess volumes, $V^{E}$, are determined from the average volume values obtained along NPT simulations for the mixtures and pure components. Similarly, excess enthalpy is obtained from the usual expression

$$
H^{E}\left(x_{2}, p, T\right)=U^{E}\left(x_{2}, p, T\right)+P V^{E}\left(x_{2}, p, T\right),
$$

and the excess internal energy, $U^{E}$, is also directly evaluated from the MD runs for the mixture and pure components. The fluctuation of the enthalpy provides a direct path for the calculation of the specific heat at constant pressure, $c_{p}^{E}$,

$$
\begin{aligned}
c_{p}\left(x_{2}, p, T\right) & =\left(\frac{\partial H\left(x_{2}, p, T\right)}{\partial T}\right)_{P} \\
& \simeq\left\langle\left(H\left(x_{2}, p, T\right)-\left\langle H\left(x_{2}, p, T\right)\right\rangle\right)^{2}\right\rangle_{N p T}
\end{aligned}
$$

and therefore,

$c_{p}^{E}\left(x_{2}, p, T\right)=c_{P}\left(x_{2}, p, T\right)-\left[x_{2} c_{p, 2}^{0}(p, T)+\left(1-x_{2}\right) c_{p, 1}^{0}(p, T)\right]$.

This property requires extremely long simulation runs, and we have assessed the validity of our results comparing the results of the fluctuation approach to those obtained by the numerical differentiation of the enthalpy with respect to temperature, for specific points.

Our results for the excess thermodynamics of our mixture system (heteronuclear model) are collected in Figure 8. The excess volume exhibits the typical volume contraction of the mixture, a characteristic of short chain alcohols. ${ }^{25,41,56}$ This is in agreement with the observed behavior in $g(r)$ (Figure 7), in which it is seen that water particles move closer to each other when the solute is incorporated.

The situation is somewhat different for the excess enthalpy. Here our model exhibits a minimum for alcoholrich solutions, in contrast with the experimental situation for methanol, ${ }^{41}$ ethanol, ${ }^{56}$ and tert-butanol. ${ }^{25}$ In these cases, the minimum occurs for water-rich conditions. Moreover, tert-butanol ${ }^{25}$ excess enthalpies change the sign as the concentration of alcohol increases but, contrary to our model's behavior, positive values occur at high alcohol concentrations. As shown by González-Salgado and co-workers, ${ }^{41}$ these discrepancies could be cured by a simple tuning of the cross interaction parameters. Even with more or less sophisticated models for pure alcohol and water, excess properties can be even qualitatively wrong when Lorentz-Berthelot mixing rules are used. ${ }^{41,56}$

Finally, in the lower graph of Figure 8 we have the excess constant pressure heat capacity, as obtained from Eq. (7). The model performance for the excess heat capacity is correlated with that of the excess enthalpy. Again here we observe the presence of a maximum in agreement with experimental results for methanol ${ }^{41}$ and tert-butanol, ${ }^{25}$ but the model predicts its position at somewhat higher concentrations of alcohol. Nonetheless, we can say that at a relatively low temperature the increase of the heat capacity reflects the 

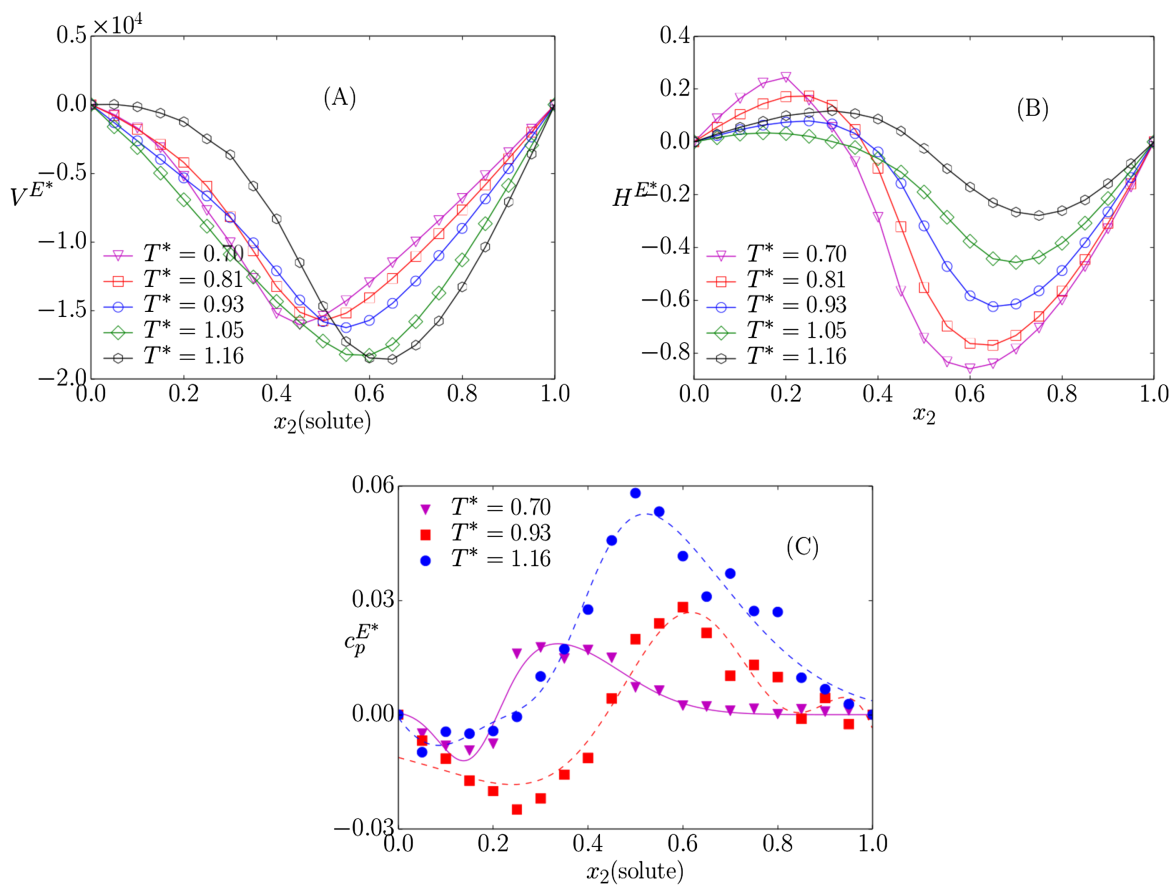

structure-making character of our solvent, in accordance with the experimental findings. Again, discrepancies such as the presence of negative values of the excess heat capacity or the shift of the maxima to regions of higher alcohol concentration can be tuned by a careful choice of the cross interaction parameters.

\section{CONCLUSIONS}

In summary, we have presented a detailed computer simulation study of a simple model for diluted alcohol-water mixtures, in which the interactions involving hydrogen bonding are represented by a two-scale potential which is known to reproduce a good number of water anomalies. Our results for the dependence of the temperature of maximum density on the solute concentration are in qualitative agreement with the experimental behavior of methanol and t-butanol solutions, whose molecules are modeled by homonuclear and heteronuclear dimers, respectively. These results indicate that for a small range of concentrations and up to certain values of pressure, these hydrogen-bonding-like solutes tend to enhance the open structures of water and hence increase the TMD, behaving as "structure-makers."

As the pressure increases, the "structure-breaker" character of the solutes is enhanced, being larger as the size of the alkyl group grows. This is understandable as the presence of the apolar group as the pressure increases makes more unfavorable the open structures which are responsible for the anomalous behavior of the model. This enthalpic effect increases with the size of the solute molecule.

Future work will focus on the dynamic anomalies (e.g., the increase of the diffusion constant with pressure) which are known to be influenced in a similar fashion when diluted hydrogen bonded solutes are present, in marked contrast with the effect of other solutes, either polar or apolar.

\section{ACKNOWLEDGMENTS}

The authors gratefully acknowledge support from the Programa Ciência sem Fronteiras do Governo Federal de Brasil, for Pesquisador Visitante Especial Grant No. 401036/2014-6. E.L. acknowledges the support from the Dirección General de Investigación Científica y Técnica under Grant No. FIS2013-47350-C5-4-R.

${ }^{1}$ J. B. Jaimes, M. Alvarez, J. V. Rojas, and R. M. Filho, Chem. Eng. Trans. 38, 139 (2014).

${ }^{2}$ M. O. Dias, T. L. Junqueira, C. E. V. Rossell, R. M. Filho, and A. Bonomi, Fuel Process. Technol. 109, 84 (2013).

${ }^{3}$ F. Franks and D. J. G. Ives, Q. Rev., Chem. Soc. 20, 1 (1966).

${ }^{4}$ G. C. Benson, P. J. D’Arcy, and O. Kiyohara, J. Solution Chem. 9, 931 (1980).

${ }^{5}$ N. C. Patel and S. I. Sandler, J. Chem. Eng. Data 30, 218 (1985).

${ }^{6}$ S. Dixit, J. Crain, W. Poon, J. Finney, and A. Soper, Nature 416, 829 (2002).

${ }^{7}$ G. S. Kell, J. Chem. Eng. Data 20, 97 (1975).

${ }^{8}$ P. Gallo, D. Corradini, and M. Rovere, J. Mol. Liq. 189, 52-56 (2014).

${ }^{9}$ L. G. Hepler, Can. J. Chem. 47, 4613 (1969).

${ }^{10}$ A. J. Darnell and J. Greyson, J. Phys. Chem. 72, 3021-3025 (1968).

${ }^{11}$ M. Tariq, J. M. S. S. Esperanca, M. R. C. Soromenho, L. P. N. Rebelo, and J. N. C. Lopes, Phys. Chem. Chem. Phys. 15, 10960 (2013).

${ }^{12}$ G. Wada and S. Umeda, Bull. Chem. Soc. Jpn. 35, 1797 (1962).

${ }^{13}$ G. Wada and S. Umeda, Bull. Chem. Soc. Jpn. 35, 646 (1962).

${ }^{14}$ F. Franks and B. Watson, Trans. Faraday Soc. 63, 329 (1967).

${ }^{15}$ M. W. Mahoney and W. L. Jorgensen, J. Chem. Phys. 112, 8910 (2000).

${ }^{16}$ I. Tomaszkiewicz and S. L. Randzio, Thermochim. Acta 103, 291 (1986).

${ }^{17} \mathrm{~J}$. Ott, G. Cornett, C. Stouffer, B. Woodfield, C. Guanquan, and J. Christensen, J. Chem. Thermodyn. 18, 867 (1986).

${ }^{18}$ M. Mundhwa and A. Henni, J. Chem. Thermodyn. 39, 1439 (2007).

${ }^{19}$ A. Ben-Naim, A. M. Navarro, and J. M. Leal, Phys. Chem. Chem. Phys. 10, 2451 (2008).

${ }^{20}$ F. Sha, T. Zhao, B. Guo, F. Zhang, X. Xie, and J. Zhang, Phys. Chem. Liq. 54, 165 (2016).

${ }^{21}$ J. Ott, J. Sipowska, M. Gruszkiewicz, and A. Woolley, J. Chem. Thermodyn. 25, 307 (1993).

${ }^{22}$ Y. Maham, T. T. Teng, L. G. Hepler, and A. E. Mather, J. Solution Chem. 23, 195 (1994).

${ }^{23}$ M. Stec, A. Tatarczuk, D. Śpiewak, and A. Wilk, J. Solution Chem. 43, 959 (2014). 
${ }^{24}$ C. G. Hanke and R. M. Lynden-Bell, J. Phys. Chem. B 107, 10873 (2003).

${ }^{25}$ D. Subramanian, J. B. Klauda, J. Leys, and M. A. Anisimov, Vestn. S.-Peterb. Univ., Ser. 4: Fiz., Khim. 4, 140 (2013).

${ }^{26}$ H. S. Frank and M. W. Evans, J. Chem. Phys. 13, 507 (1945).

${ }^{27}$ M. Nagasaka, K. M. V. Leloup, and N. Kosugi, J. Phys. Chem. B 118, 4388 (2014).

${ }^{28}$ E. Galicia-Andrés, L. Pusztai, L. Temleitner, and O. Pizio, J. Mol. Liq. 209, 586 (2015).

${ }^{29}$ S. Chatterjee, H. S. Ashbaugh, and P. G. Debenedetti, J. Chem. Phys. 123, 164503 (2005).

${ }^{30}$ M. Huš, G. Munaò, and T. Urbic, J. Chem. Phys. 141, 164505 (2014).

${ }^{31}$ M. Huš and T. Urbic, Phys. Rev. E 90, 062306 (2014).

${ }^{32}$ M. Huš, G. Žakelj, and T. Urbic, Acta Chim. Slov. 62, 524-530 (2015).

${ }^{33}$ G. Munaò and T. Urbic, J. Chem. Phys. 142, 214508 (2015).

${ }^{34}$ E. A. Jagla, Phys. Rev. E 63, 061501 (2001).

${ }^{35}$ Z. Su, S. V. Buldyrev, P. G. Debenedetti, P. J. Rossky, and H. Eugene Stanley, J. Chem. Phys. 136, 044511 (2012).

${ }^{36}$ J. L. F. Abascal and C. Vega, J. Chem. Phys. 123, 234505 (2005).

${ }^{37}$ W. L. Jorgensen, J. Phys. Chem. 90, 1276 (1986).

${ }^{38}$ S. K. Allison, J. P. Fox, R. Hargreaves, and S. P. Bates, Phys. Rev. B 71, 024201 (2005).

${ }^{39}$ A. Laaksonen, P. G. Kusalik, and I. M. Svishchev, J. Phys. Chem. A 101, 5910 (1997).

${ }^{40}$ I. Bako, T. Megyes, S. Balint, T. Grosz, and V. Chihaia, Phys. Chem. Chem. Phys. 10, 5004 (2008).
${ }^{41}$ D. González-Salgado, K. Zemánková, E. G. Noya, and E. Lomba, J. Chem. Phys. 144, 184505 (2016).

${ }^{42}$ J. N. da Silva, E. Salcedo, A. B. de Oliveira, and M. C. Barbosa, J. Chem. Phys. 133, 244506 (2010).

${ }^{43}$ M. P. Allen and D. J. Tildesley, Computer Simulation of Liquids (Clarendon Press, New York, NY, USA, 1989).

${ }^{44}$ G. S. Kell, J. Chem. Eng. Data 12, 66 (1967).

${ }^{45}$ C. A. Angell, E. D. Finch, and P. Bach, J. Chem. Phys. 65, 3063 (1976).

${ }^{46}$ J. R. Errington and P. G. Debenedetti, Nature 409, 318 (2001).

${ }^{47}$ J. D. Weeks, D. Chandler, and H. C. Andersen, J. Chem. Phys. 54, 5237 (1971).

${ }^{48}$ S. Plimpton, J. Comput. Phys. 117, 1 (1995).

${ }^{49}$ S. Nosé, J. Chem. Phys. 81, 511 (1984).

${ }^{50}$ W. G. Hoover, Phys. Rev. A 31, 1695 (1985).

${ }^{51}$ J.-P. Ryckaert, G. Ciccotti, and H. J. Berendsen, J. Comput. Phys. 23, 327 (1977).

${ }^{52}$ J.-P. Hansen and I. McDonald, Theory of Simple Liquids, 2nd ed. (Academic, London, 1990).

${ }^{53}$ C. Bores, E. Lomba, A. Perera, and N. G. Almarza, J. Chem. Phys. 143, 084501 (2015).

${ }^{54}$ H. L. Pi, J. L. Aragones, C. Vega, E. G. Noya, J. L. Abascal, M. A. Gonzalez, and C. McBride, Mol. Phys. 107, 365 (2009).

${ }^{55}$ E. Lomba, N. G. Almarza, C. Martin, and C. McBride, J. Chem. Phys. 126, 244510 (2007).

${ }^{56}$ M. Mijaković, K. D. Polok, B. Kežić, F. Sokolić, A. Perera, and L. Zoranić, Mol. Simul. 41, 699 (2014). 